\title{
Utility values associated with blindness in an adult population
}

\author{
Melissa M Brown, Gary C Brown, Sanjay Sharma, Jonathan Kistler, Heidi Brown
}

Center for

Evidence-Based

Health Care

Economics,

Flourtown, PA, USA

M M Brown

G C Brown

S Sharma

J Kistler

H Brown

Cataract and Primary Eye Care Service, Wills Eye Hospital, Jefferson Medical College, Philadelphia, PA, USA

M M Brown

Retina Vascular Unit, Wills Eye Hospital, Jefferson Medical College, Philadelphia, PA, USA

G C Brown

S Sharma

J Kistler

Departments of Ophthalmology, Epidemiology and the Cost-Effective Ocular Health Policy Unit, Queens University, Kingston, Ontario, Canada

S Sharma

Correspondence to: Melissa M Brown, Center for Evidence-Based Health Care Economics, Suite 210, 1107 Bethlehem Pike, Flourtown, PA 19031, USA

Lissa1011@aol.com

Accepted for publication 9 October 2000

\begin{abstract}
Aim-To ascertain utility values associated with varying degrees of legal blindness.

Methods-A cross sectional study on three group of patients. There were: (1) 15 patients with complete absence of vision (no light perception) in at least one eye who were asked to assume a scenario of no light perception in the second eye as well, (2) 17 patients with light perception to counting fingers in the better seeing eye, and (3) 33 patients with 20/200-20/400 vision in the better seeing eye. Utility values were measured using the time trade-off and standard gamble methods in each of the three groups.
\end{abstract}

Results-The mean time trade-off utility value for the no light perception group with the theoretical scenario of bilateral absence of light perception was $0.26(95 \%$ CI, 0.19-0.33). The mean utility value for the light perception to counting fingers group was 0.47 (95\% CI, 0.33-0.61), and the mean utility value for the 20/200-20/400 group was 0.65 (95\% CI, 0.58-0.72). Thus, patients with no light perception in one eye, who were presented with the same scenario in the second eye as well, were willing to trade almost 3 out of every 4 years of remaining life in return for perfect vision in each eye. Those with light perception to counting fingers would trade approximately 1 of 2 remaining years and those with 20/200-20/400 would trade approximately 1 of 3 remaining years.

Conclusions-There is a wide range of utility values associated with legal blindness. The utility value decreases dramatically with perceived total loss of vision (absence of light perception in each eye), compared with counting fingers to light perception vision, indicating that the preservation of even small amounts of vision in patients with legal blindness is critically important to their wellbeing and functioning in life.

(Br F Ophthalmol 2001;85:327-331)

Utility theory was developed in the 1940 s to help quantify the uncertainty that existed in varied fields. ${ }^{1}$ In the past 20 years, the application of utility theory to health care was undertaken to help quantify uncertainty regarding medical diagnostic and therapeutic procedures. ${ }^{1-3}$ In essence, the utility value of a patient is a direct reflection of patient preferences. Utility assessment evaluates how effectively a patient is able to function in his or her activities of everyday life and allows an objective measurement of quality of life associated with a health (disease) state.

By convention, a utility (or utility value) of 1.0 indicates a state of perfect health, whereas a utility of 0 indicates death. Diseases that impair quality of life minimally, such as treated systemic arterial hypertension, may cause only a minimal decrease in utility to the 0.95-0.99 range, ${ }^{2}$ whereas those that affect lifestyle dramatically, such as severe stroke, can markedly decrease the utility to $0.30 .^{4}$

Blindness has been reported by Torrance and Feeny ${ }^{2}$ to be associated with a utility of 0.39 , although the definition of blindness was not elaborated. Legal blindness of an eye is typically defined in many countries as Snellen visual acuity $\leqslant 20 / 200$ in that eye. ${ }^{5}$ Therefore, blindness could refer to vision ranging from $20 / 200$ to no light perception. It was the authors' opinion that there might be a widely varying degree of patients' perception of their quality of life within the range of vision that qualifies as legal blindness. In particular, it was believed that those with no vision whatsoever had substantially more difficulty with the daily activities of life than those with limited vision. For this reason, a study was undertaken to determine utility values associated with theoretical total blindness (no light perception in each eye) and compare them with utility values encountered with other levels of legal blindness (vision $\leqslant 20 / 200$ ) that were not as severe.

\section{Methods}

STUDY POPULATION AND SETTING

Consecutive ambulatory patients from the office practice of one physician at Wills Eye Hospital (GB) were selected for study. The patients were all adults taken predominantly from a vitreoretinal population. Included among the diseases that caused the visual loss were retinal detachment, age related macular degeneration, diabetic retinopathy, cataract, glaucoma, endophthalmitis, and central retinal vein obstruction.

Exclusion criteria for the subjects included the presence of Alzheimer's disease and other diagnosed forms of dementia. Those who either refused or were unable to answer a series of complex theoretical questions after they were asked were also excluded.

The patients were divided into three groups according to the following criteria:

(1) No light perception group. Consecutive patients with no light perception vision in at least one eye.

(2) Counting fingers to light perception group. Consecutive patients with some vision in 
each eye, but a vision of counting fingers to light perception in the better eye.

(3) 20/200-20/400 group. Consecutive patients with some vision in each eye, but a vision of 20/200-20/400 in the better eye.

DATA COLLECTION

Every person underwent a complete ophthalmological evaluation, including measurement of best corrected Snellen visual acuity. In those instances in which a pinhole vision further improved the visual acuity over the best refracted vision, the pinhole visual acuity was taken as the measured vision in the eye. Slit lamp examination and dilated fundus examination with indirect ophthalmoscopy were also performed on all patients.

Demographic information obtained at the time of ophthalmological examination included age, sex, and length of time of visual loss to the level noted at the most recent ophthalmological examination.

Study questions were administered by one of the authors (GB) in a standardised fashion using the previously described time trade-off and standard gamble methods to measure utility values. The exact wording has been reported elsewhere ${ }^{67}$ but, in essence, the time trade-off method of assessment required asking the patient how long he or she expected to live, then asking how many of those remaining years of life, if any, the patient would be willing to trade in return for a therapy that would allow his or her current vision in each eye to be transformed to permanent perfect vision (20/ $20)$ bilaterally. The proportion of years traded was then subtracted from 1.0 to yield the utility value (for example, a patient giving up 4 of 10 years would have a utility value of $1.0-0.04$ $=0.6)$. With the standard gamble method, the patient was presented with the scenario of a therapeutic technology that offered perfect vision. When the therapy worked, it worked perfectly, but the alternative result was immediate death when it did not. The patient was then asked how high a risk of death (\%), if any, he or she would be willing to accept before refusing treatment that would return the vision to $20 / 20$ in each eye. This percentage was then subtracted from 1.0 to obtain the utility value (for example, a patient willing to take up to a $30 \%$ chance of dying in return for a treatment would have a utility value of $1.0-0.3=0.7$ )

Since only one patient had no light perception vision in both eyes, all (excluding the patient with bilateral no light perception) with no light perception in one eye were questioned with the time trade-off and standard gamble methods a second time with the theoretical scenario that the vision in each eye was the same as in the worse eye (no light perception bilaterally).

\section{STATISTICAL METHODS}

The means, standard deviations, and 95\% confidence intervals were calculated for the utility values measured in the sample subgroups. The means of different sample subgroups were compared using the unpaired, heteroscedastic, two tailed Student's $t$ test.
Comparison of the means of time trade-off and standard gamble utilities within the visual stratification groups were performed using the paired, two tailed, Student's $t$ test. Statistical significance was assumed to occur at the 0.05 level.

\section{Results}

Approximately 1500 patients were screened over a 5 month period to gather the information presented herein. Among these 1500, 75 patients met the visual criteria outlines in the Methods section. Ten patients were excluded because of dementia, unwillingness, or inability to answer the questions. Thus, the results are presented on a total of 65 patients.

NO LIGHT PERCEPTION (NLP) GROUP

There were 15 patients who had no light perception vision in at least one eye. Included were nine women and six men, with an age range from 37 years to 84 years and a mean age of 61 years. One patient had no light perception in both eyes, and two had no light perception in one eye and light perception in the other eye. In the others, the vision in the second eye ranged from $20 / 20$ to counting fingers

The causes of complete loss of vision in the eyes with no light perceptions included rhegmatogenous retinal detachment in five patients, diabetic retinopathy in five patients, age related macular degeneration in two patients, glaucoma in one patient, and endophthalmitis in one patient. The mean length of time of visual loss was 4.7 years, with a range from 1 month to 13 years. Two of the patients classified as having no vision in one eye had previously undergone an enucleation.

The mean utility for this group when evaluated according to the combined vision in both eyes using the time trade-off method was 0.62 (SD $0.25 ; 95 \%$ confidence interval, 0.490.75). With the standard gamble method the mean utility was $0.73(0.31 ; 0.57-0.89)$. The difference in mean utility values using the time trade-off and standard gamble methods was not significant using a two tailed, paired $t$ test $(p=0.32)$.

When the mean utility values were calculated assuming the patients had no light perception vision in each eye, the value using the time trade-off method was 0.26 (0.14; $0.19-0.33)$. The median utility value for this time trade-off group was 0.25. For the standard gamble method the mean utility value assuming no light perception in both eyes was $0.32(0.24 ; 0.20-0.44)$. The corresponding median utility value for the standard gamble group was 0.20 . The difference between the mean utilities using the time trade-off and standard gamble groups using a paired, two tailed $t$ test was not significant $(\mathrm{p}=0.13)$. Summaries of the mean utility values for the time trade-off method are shown in Table 1, and for the standard gamble method in Table 2.

When the mean utility value with the current vision in each eye using the time trade-off method (0.62) and the mean utility assuming 
Table 1 Time trade-off utility values for the NLP, LP-CF, and 20/200-20/400 groups

\begin{tabular}{llllll}
\hline Group & Mean utility & $S D$ & $95 \% C I$ & $p$ Value $^{\star}$ & V Value $^{\star *}$ \\
\hline NLP & 0.26 & 0.08 & $0.19-0.33$ & NA & 0.007 \\
LP-CF & 0.47 & 0.29 & $0.33-0.61$ & 0.007 & NA \\
$20 / 200-20 / 400$ & 0.65 & 0.21 & $0.58-0.72$ & $0.6 \times 10^{-12}$ & 0.03 \\
\hline
\end{tabular}

NLP $=$ no light perception in each eye LP-CF $=$ light perception to counting fingers in the better eye; $20 / 200-20 / 400=20 / 200$ to $20 / 400$ in the better eye; ${ }^{\star}$ using the heteroscedastic Student's $t$ test compared with the NLP group; ${ }^{\star \star}$ using the heteroscedastic Student's $t$ test compared with the light perception to counting fingers group; NA = not applicable.

Table 2 Standard gamble utility values for the NLP, LP-CF, and 20/200-20/400 groups

\begin{tabular}{llllll}
\hline Group & Mean utility & $S D$ & $95 \% C I$ & $p$ Value $^{\star}$ & $p$ Value $^{\star *}$ \\
\hline NLP & 0.32 & 0.24 & $0.20-0.44$ & NA & 0.003 \\
LP-CF & 0.60 & 0.29 & $0.46-0.74$ & 0.003 & NA \\
20/200-20/400 & 0.80 & 0.21 & $0.73-0.87$ & $0.3 \times 10^{-10}$ & 0.02
\end{tabular}

NLP = no light perception in each eye $\mathrm{LP}-\mathrm{CF}=$ light perception to counting fingers in the better eye; $20 / 200-20 / 400=20 / 200$ to $20 / 400$ in the better eye; ${ }^{\star}$ using the heteroscedastic Student's $t$ test compared with the NLP group; ${ }^{\star}$ using the heteroscedastic Student's $t$ test compared with the light perception to counting fingers group; $\mathrm{NA}=$ not applicable.

no light perception in each eye using the time trade-off method (0.26) were compared, there was a significant difference using the paired, two tailed $t$ test $(\mathrm{p}=0.0001)$. With the standard gamble method, the difference between the mean of utility values for current vision (0.60) versus that assuming bilateral no light perception (0.32) was also significant $(p=0.0001)$.

Among the 15 patients in this group, five had visual loss to their present level at the time of study evaluation for 1 year or less. The mean utility for this group, assuming the no light perception in both eyes scenario and using the time trade-off method was 0.17 . For the 10 patients who had visual loss to the present level for greater than 1 year, the mean utility, assuming no light perception vision in both eyes and using the time trade-off method, was 0.31 . The means between the 1 year or less group and the greater than 1 year group were statistically significant, using the heteroscedastic, unpaired, two tailed $t$ test $(\mathrm{p}=0.02)$.

\section{LIGHT PERCEPTION TO COUNTING FINGERS GROUP}

Patients in this group had bilateral sight and a vision of light perception to counting fingers in the better eye. There were 17 patients, with a mean age of 72 years and an age range of 46-81 years. The causes of visual loss were age related macular degeneration in eight patients, diabetic retinopathy in five patients, central retinal vein occlusion in two patients, glaucoma in one patient, and retinal detachment in one patient. Two patients had light perception in the better eye and 15 had counting fingers to hand motion vision in the better seeing eye.

The mean utility for this group using the time trade-off method (Table 1) was 0.47 (SD 0.29 ; 95\% CI $0.33-0.61$ ) and the median utility value was 0.50 . With the standard gamble method (Table 2 ), the mean utility value was $0.60(0.29 ; 0.46-0.74)$ and the median utility value was 0.60 . The difference between the mean utilities derived from the time trade-off and standard gamble groups using a paired, two tailed $t$ test was not significant ( $\mathrm{p}=0.57$ ).

There was a significant difference in time trade-off mean utility values between the NLP
Table 3 Comparison of mean time trade-off and standard gamble utility values

\begin{tabular}{llll}
\hline Group & TTO utility & SG utility & p Value \\
\hline NLP & 0.26 & 0.32 & 0.13 \\
LP-CF & 0.47 & 0.60 & 0.57 \\
20/200-20/400 & 0.65 & 0.80 & 0.03 \\
\hline
\end{tabular}

TTO = time trade-off method; SG = standard gamble method; NLP = no light perception; LP = light perception; CF = counting fingers; $p$ value comparing the TTO and SG methods within each visual group using the paired, two tailed Student's $t$ test.

group and the light perception to counting fingers group using the unpaired, two tailed, heteroscedastic $t$ test $(\mathrm{p}=0.007)$. The difference in means between the two groups using the standard gamble method was also significant $(p=0.003)$

Eight patients had visual loss for 1 year or less, while nine had visual loss for greater than 1 year. The mean time trade-off utility value in those with visual loss for 1 year or less was 0.46 , while in those with visual loss for greater than 1 year it was 0.48 . The difference in these utility means was not significant using the unpaired, two tailed, heteroscedastic $t$ test $(\mathrm{p}=$ 0.89).

\section{0/200 TO 20/400 GROUP}

Patients in this group had bilateral sight and a vision of $20 / 200$ to $20 / 400$ in the better eye. The group consisted of 33 patients with a mean age of 69 years and a range 37-84 years. The causes of visual loss included age related macular degeneration in 14 patients, diabetic retinopathy in 13 patients, retinal vein occlusion in three patients, and retinal detachment, glaucoma and cataract in one patient each.

The mean utility with the time trade-off method for this group was 0.65 (SD 0.21 ; CI, $0.58-0.72$ ) and the median utility value was 0.67 . With the standard gamble method, the mean utility was $0.80(0.21 ; 0.73-0.87)$ and the median utility was also 0.80 . The difference between the mean utilities derived from the time trade-off and standard gamble groups using a paired, two tailed $t$ test was significant $(p=0.03)$. A summary of the different time trade-off and standard gamble mean utility values for the three visual groups is shown in Table 3.

When this sample subgroup was divided into those patients who had visual loss to the present level for 1 year or less versus greater than 1 year, 12 patients were in the former group and 21 in the latter group. The mean utility for those with visual loss for 1 year or less was 0.58 , whereas the mean utility for those with visual loss for greater than 1 year was 0.69 . The difference in mean utilities was not significant using the paired, two tailed $t$ test $(\mathrm{p}=0.21)$

There was a highly significant difference in the mean utility values, employing the time trade-off method (Table 1), between the NLP group and the 20/200-20/400 group using the two tailed, heteroscedastic $t$ test ( $\mathrm{p}=0.6 \times$ $10^{-12}$ ). With the standard gamble method (Table 2), there was also a significant difference in the mean utility values between the NLP and 20/200-20/400 groups $(p=0.3 \times$ 
$\left.10^{-10}\right)$. A comparison of mean utility values between the light perception to counting fingers and the 20/200-20/400 groups revealed a significant difference with both the time trade-off method $(\mathrm{p}=0.03)$ and the standard gamble method $(\mathrm{p}=0.02)$ (Tables 1 and 2$)$.

\section{Discussion}

Von Neumann and Morgenstern are generally given credit for developing utility theory in the 1940 s to attempt to quantify uncertainty. ${ }^{1}$ Since that time, it has also been employed in the healthcare arena to help to objectively quantify uncertainty. ${ }^{1-4} 6-8$

The importance of utility values lies in the fact that they provide an objective measure of patient preferences of how valuable a function such as vision is to the patient from the patient's point of view. Increasing numbers of authors $^{8-10}$ believe, as do the authors of this article, that patient preferences are key to decisions that affect their health care.

Using the time trade-off method with data from the general public, Torrance and Feeny ${ }^{2}$ noted that blindness yielded a utility of 0.39 . Nevertheless, their definition of blindness was not quantified. Our data correlate somewhat with those of Torrance and Feeny, ${ }^{3}$ but further demonstrate that assigning one utility value to blindness is inadequate. On the contrary, there appears to be a wide range of utilities associated with different levels of vision within the range of legal blindness (visual acuity $\left.\leqslant 20 / 200^{5}\right)$. The worse the vision in the better eye, the lower the utility. And the difference can be dramatic. While those in the 20/200-20/ 400 group had a mean time trade-off utility of 0.65 , those in the counting fingers to hand motions group had a corresponding value of 0.47 and those in the NLP group a mean utility of 0.26 .

The mean utility values of the NLP group and the light perception to counting fingers and 20/200-20/400 groups were all significantly different, as were the mean utility values of the light perception to counting fingers and 20/200-20/400 groups. These differences occurred with both the time trade-off and standard gamble methodologies. There was also negligible overlap in the $95 \%$ confidence intervals of the groups with both the time trade-off and standard gamble methods, thereby strongly suggesting that the visual groups in the study are objectively quantifiable and distinctly different with regard to patient preferences and the ability to function in the activities of daily life.

A number of different methods have been developed to measure utility values, but the time trade-off and standard gamble methods are the most widely employed. ${ }^{2} 7$ The standard gamble method has been accepted as the "gold standard" for the measurement of utility values, but some authors question its validity. ${ }^{11} 12$ We noted a statistically significant difference in mean utility values using the time trade-off and standard gamble methods for one of our three visual subgroup samples, the
20/200-20/400 group. There was no significant difference, however, with the no light perception or 20/200-20/400 groups. We cannot say with certainty which method is preferable, but it is the unanimous subjective opinion of the present authors that patients have more difficulty understanding the standard gamble concept. The fact that most of the patients have never faced the possibility of immediate death might make quantifying the percentage of risk of imminent death with the standard gamble scenario quite difficult. Many patients appeared to be repulsed by the thought of immediate death, and thus emotion probably has a greater role with the standard gamble methodology. The standard gamble method has been shown to overestimate risk aversion compared with other methods, and that was certainly the case in the present paper. ${ }^{11}$ For each of the three visual acuity stratifications, the mean standard gamble utility value was higher than the corresponding time trade-off value. Of probable greater importance, however, than deciding whether to use the time trade-off method or the standard gamble method, is the concept that utility values should be compared using the same method when contrasting utilities associated with different health states.

Utilities, or utility values, have been developed for a number of health states that affect quality of life. ${ }^{2}$ Among these are mild angina, utility of 0.90 ; moderate angina, utility of 0.70 ; severe angina, utility of 0.50 . Home dialysis yields a utility of approximately 0.54 and severe depression a utility of 0.45 .

Our interpretation of the mean utilities we found indicates that patients value vision very highly. Surprising to us was the marked difference in mean utility between those who are just over the line of legal blindness (20/200-20/400 group) and those who are totally blind (NLP group). The marked difference in utility findings between these two groups suggests that patients with even limited vision very highly value what vision they have remaining, despite the appearance to a casual outside observer that it may be minimal. Losing all light perception in each eye is exceptionally undesirable, falling between the disabilities caused by a moderate stroke state (utility = 0.39) to severe stroke state (utility $=0.12) .{ }^{4}$ It suggests to the ophthalmologist that trying to preserve as much vision as possible, even if the patient is legally blind, is very important to help maintain a person's quality of life.

It is of note that the length of time of visual loss affects the utility value. We found a statistically significant difference in utility value in the no light perception group between those in our study who had visual loss to the present level for 1 year or less, as versus for greater than 1 year. We have also noted this in other populations. ${ }^{13}$ The conclusion that can be drawn from these observations is that patients can learn to accommodate to their visual disabilities, and can improve their coping mechanisms with time. One would most likely expect an otherwise healthy child with bilateral no light 
perception vision due to retinopathy of prematurity in both eyes to have less difficulty functioning in life's everyday activities than a 70 year old who suddenly develops no light perception vision in each eye secondary to giant cell arteritis.

As is the case with visual disability, a person can also accommodate to other disability states with time. ${ }^{7}$ Of importance, therefore, is the thought that, when comparing utilities across different disease states, thought should be given to the length of time the disease has been present at the current degree of impairment. Comparing utilities in a sample group with relatively acute changes may be misleading if likened to utilities from a sample group with a substantially more chronic disease process.

The majority of patients in our study had visual loss occurring secondary to vitreoretinal diseases. While a possible weakness of the study, it has been shown that visual loss associated with macular degeneration is comparable by utility measurement with that seen with diabetic retinopathy or cataract. ${ }^{13}$ It appears to be the degree of visual loss in the better seeing eye, rather than the underlying pathophysiological process causing the visual loss, that is most directly correlated with a utility value. ${ }^{13}$

One possible flaw in our data is the fact that in the NLP group, only one patient truly had no light perception vision in each eye. Finding a group with visual loss to the level of incontrovertible no light perception in each eye is extremely difficult. We therefore selected what we believe to be an adequate substitute, a group comprising patients who have experienced no light perception in one eye and can therefore most probably appreciate better than others the consequences of having a similar state bilaterally. It has been demonstrated that patients with visual loss in one eye can very accurately estimate the disability that would occur if the vision was to be lost to a similar degree in the second eye. ${ }^{14}$ Additionally, the patients with no light perception in one eye had a tight $95 \%$ confidence interval with the bilateral no light perception scenario (mean utility 0.26 ; 95\% CI, 0.19-0.32) suggesting, high reproducibility within this group. The relatively tight interval using the standard gamble theory as well (mean utility $0.32 ; 95 \%$ CI, $0.20-0.44$ ), also confirms the reproducibility of the bilateral no light perception scenario in the NLP group.

Utilities have previously been measured using a number of different groups other than patients, including using physicians' perceptions of what the utilities should be, administrators' perceptions, and the perceptions of the general public. ${ }^{2}$ The authors here believe, as do others, ${ }^{8-10}$ that patient perceptions are the most valid for judging their diminution of quality of life induced by a disease state that they have experienced first hand.

Utility values quantitate patient preferences and provide an objective measure of the degree of disability imposed upon a patient by a disease process. In essence, they allow a measure of the quality of life associated with a health state. Consequently, an improvement in utility values can be used to objectively measure the degree of efficacy of an interventional medical therapy in improving a patient's quality of life. When employed in combination with tenets of evidence based medicine, such as clinical trials and meta-analysis, ${ }^{15}$ as well as with decision analysis using Markov modelling, ${ }^{16}$ utilities facilitate objective measurement of cost effectiveness. These measurements of cost effectiveness allow the comparison of vastly different disease state treatments, and can be used to objectively quantitate the value to individual patients and to society of diagnostic and therapeutic medical interventions. ${ }^{141718}$

Supported in part by the Retina Research and Development Foundation, Philadelphia, USA

1 Weinstein MC, Stasson WB. Foundations of costeffectiveness analysis for health and medical practices. $N$ Engl f Med 1977;296:716-21.

2 Torrance GW, Feeny D. Utilities and quality-adjusted life years. Int $\mathcal{F}$ Technology Assessment in Health Care, 1989;2: 559-75.

3 Froberg DG, Kane RL. Methodology for measuring healthstate preferences-II. Scaling methods. F Clin Epidemiol $1989 ; 42: 459-71$

4 Lee TT, Solomon NA, Heidenreich PA, et al. Costeffectiveness of screening for carotid stenosis in asymptomatic patients. Ann Intern Med 1997;126:337-46.

5 Newell FW. Ophthalmology. Principles and concepts. 6th ed. St Louis: CV Mosby, 1986:149.

6 Brown MM, Brown GC, Sharma S, et al. Utility values and diabetic retinopathy. Am $\mathcal{F}$ Ophthalmol 1999;126:327-30.

7 Redelmeier DA, Detsky AS. A clinician's guide to utility measurement. Med Decis Making 1995;22:271-80.

8 Kassirer JP. Adding insult to injury: usurpng patients' prerogatives. N Engl F Med 1983;308:898-901.

9 Angell M. Patients' preferences in randomized clinical trials. N Engl f Med 1984;310:1385-7.

10 Kassirer JP. Incorporating patients' preferences into medical decisions. N Engl f Med 1994;380:1895-986.

11 Wakker P, Stiggelbout A. Explaining distortions in utility elicitation through the rank-dependent model for risky choices. Med Decis Making 1995;15:180-6.

12 Richardson J. Cost utility analysis: what should be measured. Soc Sci Med 1994;39:7-21.

13 Brown GC. Vision and quality of life. Trans Am Ophthalmol Soc 1999;92:474-511.

14 Brown GC, Brown MM, Sharma S, et al. Patient perceptions of bilateral visual loss. A utility value analysis. perceptions of bilateral visual lo

15 Sharma S. Levels of evidence and interventional ophthalmology. Can f Ophthalmol 1997;32:359-62.

16 Sonnenberg FA, Beck JR. Markov models in medical decision making: A practical guide. Med Decis Making 1993;13:322-38.

17 Brown GC, Sharma S, Brown MM, et al, Evidenced-based medicine and cost-effectiveness. F Healthcare Fin 1999;26: 14-23.

18 Brown MM, Brown GC, Sharma S, et al. Evidence-based medicine, utilities and quality of life. Curr Opin Ophthalmol 1999;10:221-6. 